\section{Endoscopic intracavitary pull-through vacuum treatment of an insufficient pancreaticogastrostomy}

A 73-year-old patient underwent pyloruspreserving pancreatic head resection due to a branch-duct type intraductal papillary mucinous neoplasm. At Day 7, repeat laparotomy was performed because of elevated amylase concentrations in the abdominal drains. During the procedure, signs of pancreatitis were observed but no pancreaticogastrostomy leak was seen. Drains provided continuous irrigation. At Day 9 gastric liquid was observed in the drains and 5 days later a computed tomography scan confirmed insufficiency of the pancreaticogastrostomy. As the pa- tient was in a stable clinical condition, continuous irrigation was continued. On endoscopic placement of a jejunal feeding tube at Day 40, broad pancreaticogastrostomy insufficiency with an infected cavity was seen, with visible abdominal drainage ( $\bullet$ Fig. 1$)$.

Endoscopic vacuum treatment was initiated. A gastric tube was connected to the abdominal drain at its external end, and the drain was then grasped endoscopically in the cavity and drawn out orally. An endosponge (Endo-SPONGE; B-Braun Melsungen AG, Melsungen, Germany) was minimized in size, connected to the gastric tube ( $\bullet$ Fig.2a), and drawn into the cavity under endoscopic view by pulling the gastric tube ( $\mathbf{F i g . 2 b}$ ). The vacuum pump applying a negative pressure of $30 \mathrm{mmHg}$ was then connected to the sponge and the sponge was changed twice (at 3-day intervals). The endosponge had a thread attached to enable it to be easily grasped for exchange later. At 8 days after initial sponge placement, a clean cavity with sponge-induced granulation tissue was observed ( $\bullet$ Fig.3). A rubber drain was introduced into the cavity as described above ( $\bullet$ Fig. 4 ) in order to induce tissue granulation and promote cavity healing. The rubber drain was drawn back slowly over 17 days. At 26 days after beginning endoscopic treatment, the anastomotic leak and cavity were completely healed ( $\bullet$ Fig. $\mathbf{5}$ ).

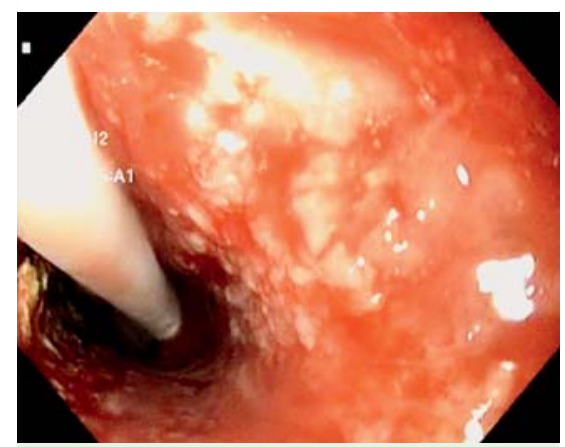

Fig. 3 The clean cavity with granulation tissue after vacuum treatment.

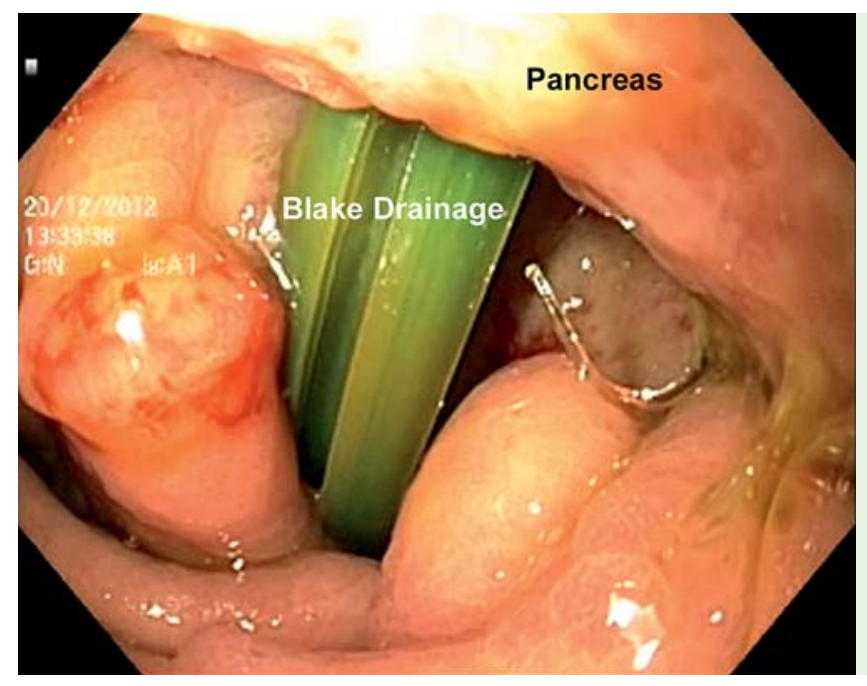

Fig. 1 View into the infected cavity with visible abdominal drainage.
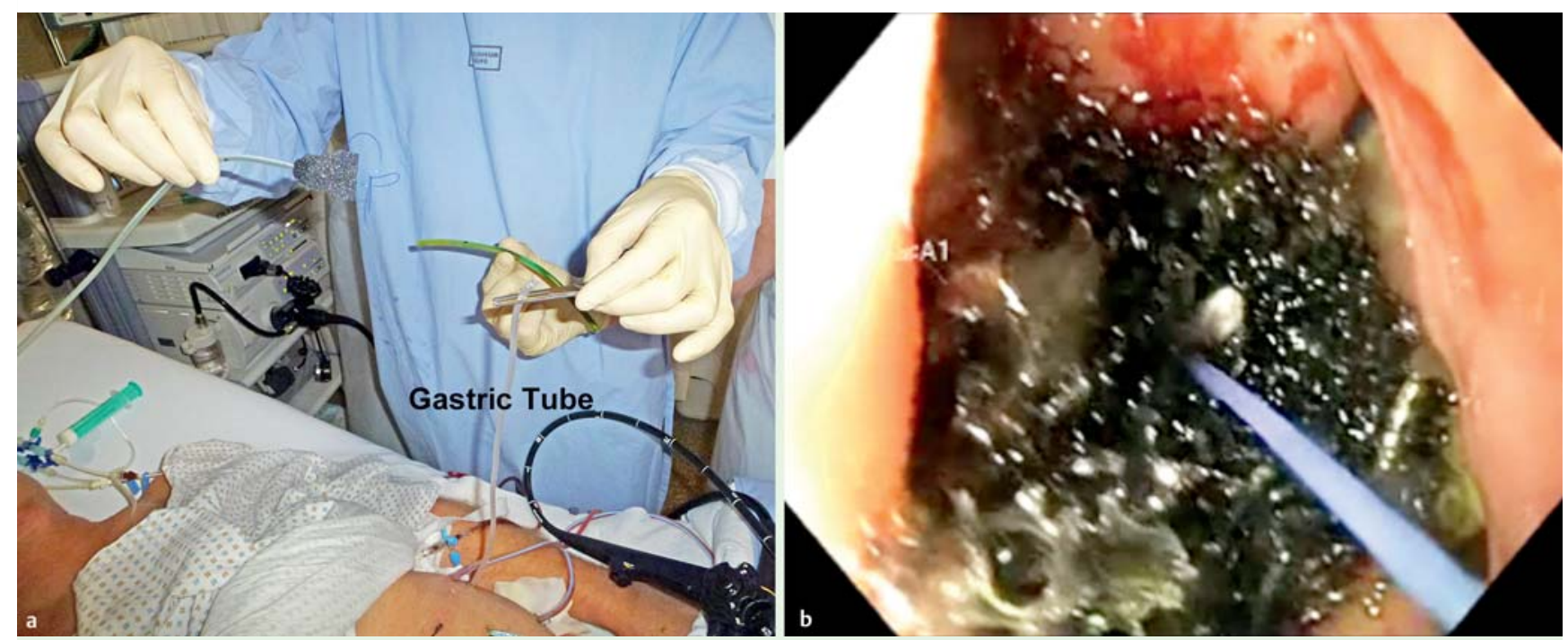

Fig.2 Endosponge placement. a The endosponge was connected to a gastric tube prior to the pull-through manuever. $\mathbf{b}$ The endosponge was drawn into the cavity; a blue thread attached to the sponge enabled grasping for sponge exchange. 


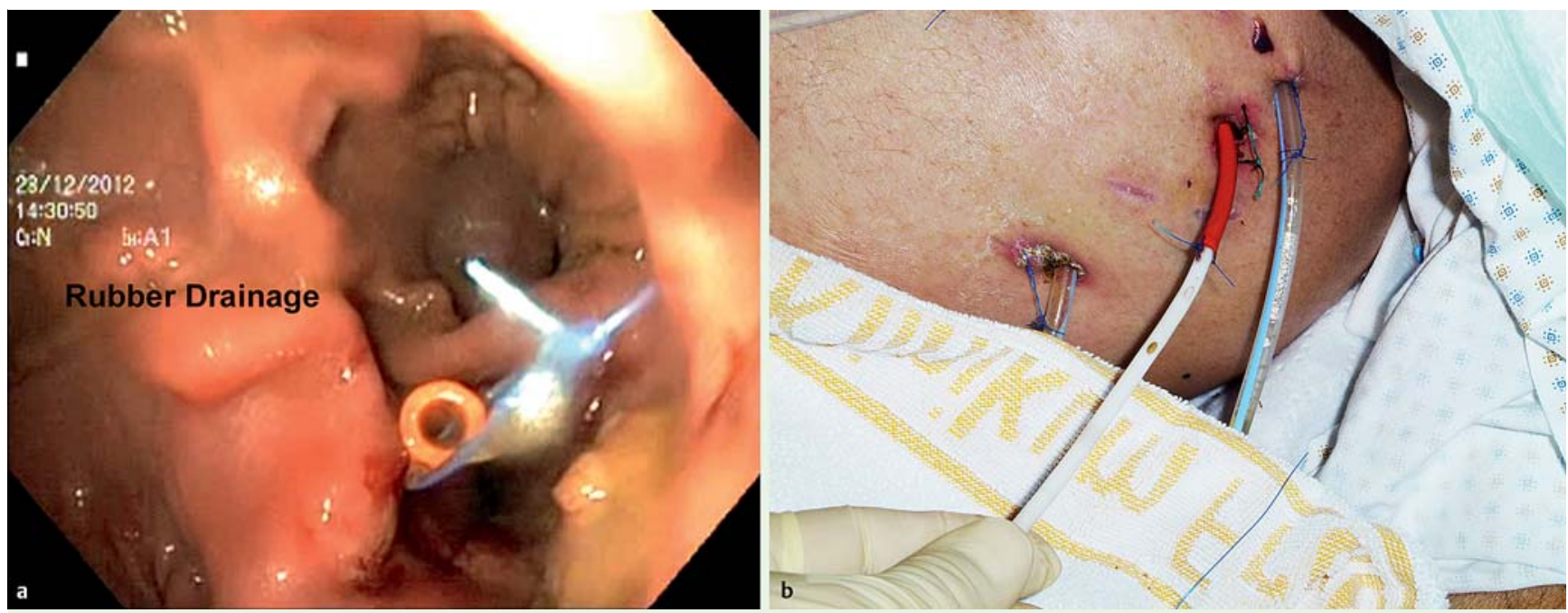

Fig. 4 Drain placement to promote healing. a A rubber drain connected to a blue thread was drawn into the cavity. $\mathbf{b}$ The rubber drain (red) after the pullthrough maneuver, still connected to the gastric tube (white).

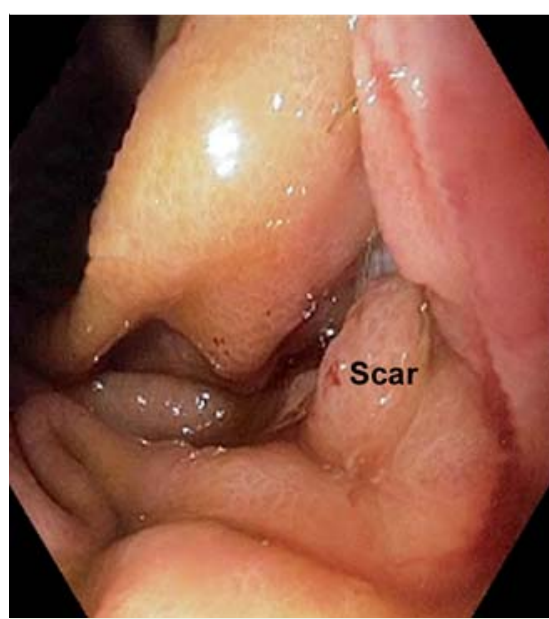

Fig. 5 Residual scar of the insufficient pancreaticogastrostomy.

Relevant co-morbidities were type 2 diabetes mellitus treated with metformin $2 \times$ $1 \mathrm{~g} /$ day and coronary heart disease. During the whole treatment period prior to the endoscopic intervention, the patient received pantoprazol $40 \mathrm{mg} /$ day and antibiotics as deemed appropriate according to the resistogram of micro-organisms found in the abdominal drains (cefuroxime, metronidazole, fluconazole, dapto- mycin, imipenem). The antibiotic treatment was stopped 3 days after placement of the first endosponge because abdominal smears became sterile and signs of systemic inflammatory response disappeared. Follow-up after 11 months revealed a symptom-free patient.

Insufficiency of a pancreaticogastrostomy is difficult to treat because simple oversewing of the leak generally fails. Schorsch et al. [1] first reported successful treatment of an insufficient pancreaticogastrostomy by intraluminal endoscopic vacuum treatment. Unlike Schorsch et al., we chose intracavitary endosponge placement by endoscopic pull-through. In our opinion, intracavitary sponge placement is preferable, but it is sometimes difficult to introduce the sponge into the cavity with the endoscope due to a narrow opening or lack of space in the cavity. Therefore, in cases with visible percutaneous abdominal drainage in the infected cavity, endoscopic vacuum treatment using a pull-through manuever is a good treatment option.

\section{Endoscopy_UCTN_Code_TTT_1AT_2AF}

\section{Competing interests: None}

\section{Andreas Fischer, Hans Juergen Richter-Schrag, Jens Hoeppner, Alexander Braun, Stefan Utzolino}

Department of General and Visceral Surgery, Albert-Ludwigs University of Freiburg - Surgical Endoscopy, Freiburg, Germany

\section{References}

1 Schorsch T, Müller C, Loske G. Pancreaticogastric anastomotic insufficiency successfully treated with endoscopic vacuum therapy. Endoscopy 2013; 45 (Suppl. 02): E141-142

\section{Bibliography}

DOI http://dx.doi.org/

10.1055/s-0034-1364951

Endoscopy 2014; 46: E218-E219

(c) Georg Thieme Verlag KG

Stuttgart · New York

ISSN 0013-726X

Corresponding author

\section{Andreas Fischer, MD}

Department of General and Visceral Surgery

Albert-Ludwigs University of Freiburg -

Surgical Endoscopy

Hugstetter Str. 55

79106 Freiburg

Germany

Fax: +49-761-2702543

andreas.fischer@uniklinik-freiburg.de 